Preprint from http://www.thephilosophyofinformation.net

This paper has been accepted for publication in

The Information Society (Taylor and Francis)

Permission to make digital or hard copies of all or part of this work for personal or classroom use is granted without fee provided that copies are not made or distributed for profit or commercial advantage and that copies bear this notice and the full citation on the first page. To copy otherwise, or republish, to post on servers or to redistribute to lists, requires prior specific permission and/or a fee.

It is a publisher's requirement to display the following notice:

The documents distributed by this server have been provided by the contributing authors as a means to ensure timely dissemination of scholarly and technical work on a noncommercial basis. Copyright and all rights therein are maintained by the authors or by other copyright holders, notwithstanding that they have offered their works here electronically. It is understood that all persons copying this information will adhere to the terms and constraints invoked by each author's copyright. These works may not be reposted without the explicit permission of the copyright holder.

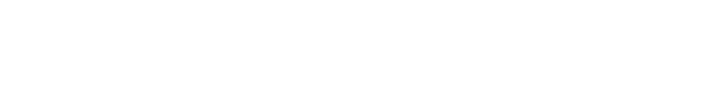

$\mathrm{o}=$ University of Oxford, email=luciano.

Floridi

floridi@philosophy.oxford.ac.uk

Reason: I am the author of this

document

Date: 2006.09.01 16:44:26 +01'00'
} 


\title{
A look into the future impact of ICT on our lives
}

\section{Luciano Floridi}

Dipartimento di Scienze Filosofiche, Università degli Studi di Bari; Faculty of Philosophy and IEG, OUCL, University of Oxford.

Address for correspondence: St Cross College, OX1 3LZ, Oxford, UK;

luciano.floridi@philosophy.oxford.ac.uk

\begin{abstract}
This paper may be read as a sequel of a 1995 paper, published in this journal, in which I predicted what sort of transformations and problems were likely to affect the development of the Internet and our system of organised knowledge in the medium term. In this second attempt, I look at the future developments of Information and Communication Technologies and try to guess what their impact on our lives will be. The forecast is that, in information societies, the threshold between online and offline will soon disappear, and that once there won't be any difference, we shall become not cyborgs but rather inforgs, i.e. connected informational organisms.
\end{abstract}

\section{Keywords}

Cyborgs; digital environment; inforgs; infosphere; IT-entities; re-ontologization. 
If you can look into the seeds of time, And say which grain will grow and which will not, Speak then to me, who neither beg nor fear Your favours nor your hate. Shakespeare, Macbeth, Act I, Scene III, 59-62.

\section{Digital seeds}

In 1995, I was invited to give a keynote speech at the UNESCO headquarters in Paris, to celebrate the fiftieth anniversary of the organisation. On that occasion, I was asked to predict what sort of transformations and problems were likely to affect the development of the Internet and our system of organised knowledge in the medium term. The challenge turned into an article, a synthesis of which was published in this journal (Floridi [1996]).

They say there are only two kinds of predictions: wrong and lucky. Mine was lucky, and so I thought I might tempt fate once more. This time, however, I shall not be concerned with the system of organised knowledge. Rather, I shall focus, more generally, on future developments in ICTs and their impact on our lives. And since there would be no merit in predicting the obvious, I will avoid issues such as rising concerns about privacy and identity theft, spamming, viruses, or the importance of semantic tagging, online shopping and virtual communities. Nor will I try to steal ideas from those who know better than I do the future development of the actual technologies (see for example O'Reilly [2005], Microsoft Research [2005], Nature [2006]). I will, instead, stick to what philosophers do better, conceptual analysis, and seek to capture the new Weltanshaung that might be dawning on us.

\section{Digital ICTs as re-ontologizing technologies}

In order to grasp the ICT scenarios that we might witness and experience in the near future, it is useful to introduce two key-concepts at the outset, those of "infosphere" and of "reontologization”.

Infosphere is a neologism I coined years ago on the basis of "biosphere", a term referring to that limited region on our planet that supports life. It denotes the whole informational environment constituted by all informational entities (thus including informational agents as well), their properties, interactions, processes and mutual relations. It is an environment 
comparable to, but different from cyberspace (which is only one of its sub-regions, as it were), since it also includes off-line and analogue spaces of information. We shall see that it is also an environment (and hence a concept) that is rapidly evolving.

Re-ontologizing is another neologism that I have recently introduced in order to refer to a very radical form of re-engineering, one that not only designs, constructs or structures a system (e.g. a company, a machine or some artefact) anew, but that fundamentally transforms its intrinsic nature. In this sense, for example, nanotechnologies and biotechnologies are not merely re-engineering but actually re-ontologizing our world.

Using the two previous concepts, my basic claim can now be formulated thus: digital ICTs are re-ontologizing the very nature of (and hence what we mean by) the infosphere, and here lies the source of some of the most profound transformations and challenging problems that our information societies will experience in the close future, as far as technology is concerned.

In the rest of this article, I mean to clarify and substantiate this simple claim by highlighting three fundamental trends in the re-ontologization of the infosphere and some of their significant implications.

\section{The rise of the frictionless infosphere}

The most obvious way in which the new ICTs are re-ontologizing the infosphere concerns (a) the transition from analogue to digital data and then (b) the ever-increasing growth of our digital space. Both phenomena are very familiar and require no explanation, but a brief comment may not go amiss.

In their second study on information storage and flows, Lyman and Varian [2003] write that "Print, film, magnetic, and optical storage media produced about 5 exabytes of new information in 2002. Ninety-two percent of the new information was stored on magnetic media, mostly in hard disks. [...] Five exabytes of information is equivalent in size to the information contained in 37,000 new libraries the size of the Library of Congress book collections.” Although the production of analogue data is still increasing, the infosphere is becoming more digital by the day. Paraphrasing Motoko Kusanagi, the infosphere is now vast and infinite (Kokaku Kidotai, Ghost in the Shell, 1995). A simple example may help to drive the point home: the new Large Hadron Collider that is being built at the CERN (http://lhc.web.cern.ch/lhc/) to 
explore the physics of particles will produce about 1.5GB data per second, or about 10 petabytes of data annually, a quantity of data a thousand times larger than the Library of Congress's print collection and at least twice as large as Google's whole data storage, reported to be approximately 5 petabytes (Mellor [April 06, 04]).

This radical re-ontologization of the infosphere is largely due to the fundamental convergence between digital resources and digital tools. The ontology of the information technologies available (e.g. software, databases, communication channels and protocols etc.) is now the same as (and hence fully compatible with) the ontology of their objects. This was one of Turing's most consequential intuitions: in the re-ontologized infosphere, there is no longer any substantial difference between the processor and the processed, so the digital deals effortlessly and seamlessly with the digital. This potentially eliminates one of the most long-standing bottlenecks in the infosphere and, as a result, there is a gradual erasure of ontological friction.

Ontological friction refers to the forces that oppose the flow of information within (a region of) the infosphere, and hence (as a coefficient) to the amount of work and effort required to generate, obtain, process and transmit information in a given environment, e.g. by establishing and maintaining channels of communication and by overcoming obstacles in the flow of information such as distance, noise, lack of resources (especially time and memory), amount and complexity of the data to be processed, and so forth. Given a certain amount of information available in (a region of) the infosphere, the lower the ontological friction in it, the higher the accessibility of that amount of information becomes. Thus, if one quantifies ontological friction from 0 to 1 , a fully successful firewall would produce a 1.0 degree of friction, i.e. a complete standstill in the flow of information through its "barrier". On the other hand, we describe our society as informationally porous the more it tends towards a 0 degree of informational friction.

Because of their "data superconductivity”, ICTs are well-known for being among the most influential factors that affect the ontological friction in the infosphere. We are all acquainted with daily aspects of a frictionless infosphere, such as spamming and micrometering. Three other significant consequences are:

a) no right to ignore: in an increasingly porous society, it will become progressively less credible to claim ignorance when confronted by easily predictable events (e.g. as George W Bush did 
with respect to Hurricane Katrina's disastrous effects on New Orleans's flood barriers) and hardly ignorable facts (e.g. as Tessa Jowell did with respect to her husband's finances); and b) vast common knowledge: this is a technical term from epistemic logic, which basically refers to the case in which everybody not only knows that $p$ but also knows that everybody knows that everybody knows that $p$. In other words, (a) will also be the case because metainformation about how much information is, was or should have been available will become overabundant.

From (a) and (b) it follows that, in the future,

c) we shall witness a steady increase in agents' responsibilities. ICTs are making humanity increasingly accountable, morally speaking, for the way the world is, will and should be (Floridi and Sanders [2001]).

\section{The global infosphere or how information is becoming our ecosystem}

During the last decade or so, we have become accustomed to conceptualising our life online as a mixture between an evolutionary adaptation of human agents to a digital environment, and a form of post-modern, neo-colonization of the latter by the former. This is probably a mistake. ICTs are as much re-ontologising our world as they are creating new realities. The threshold between here (analogue, carbon-based, off-line) and there (digital, silicon-based, online) is fast becoming blurred, but this is as much to the advantage of the latter as it is of the former. Adapting Horace’s famous phrase, “captive cyberspace is conquering its victor”.

The digital is spilling over into the analogue and merging with it. This recent phenomenon is variously known as "Ubiquitous Computing”, “Ambient Intelligence”, “The Internet of Things" (ITU report, November 2005 www.itu.int/internetofthings) or "Webaugmented things”. It is or will soon be the next stage in the digital revolution.

Basically, the increasing digital re-ontologization of artefacts and of whole (social) environments suggests that soon it will be difficult to understand what life was like in predigital times (to someone who was born in 2000 the world will always have been wireless, for example) and, in the near future, the very distinction between online and offline will become blurred and then disappear. To put it dramatically, the infosphere is progressively absorbing any other space. Let me explain. 
In the (fast approaching) future, more and more objects will be ITentities able to learn, advise and communicate with each other. A good example (but it is only an example) is provided by RFID (Radio Frequency IDentification) tags, which can store and remotely retrieve data from an object and give it a unique identity, like a barcode. Tags can measure $0.4 \mathrm{~mm}^{2}$ and are thinner than paper. Incorporate this tiny microchip in everything, including humans and animals, and you have created ITentities. This is not science fiction. According to a report by Market Research Company InStat, the worldwide production of RFID will increase more than 25-fold between 2005 and 2010 and reach 33 billion. Imagine networking these 33 billion ITentities together with all the hundreds of millions of PCs, DVDs, iPods, and ICT devices available and you see that the infosphere is no longer "there" but "here" and it is here to stay. Your Nike and iPod already talk to each other (http://www.apple.com/ipod/nike/).

Nowadays, we are used to considering the space of information as something we log-in to and log-out from. Our view of the world (our metaphysics) is still modern or Newtonian: it is made of "dead" cars, buildings, furniture, clothes, which are non-interactive, irresponsive and incapable of communicating, learning, or memorizing. But what we still experience as the world offline is bound to become a fully interactive and responsive environment of wireless, pervasive, distributed, $a 2 a$ (anything to anything) information processes, that works $a 4 a$ (anywhere for anytime), in real time. This will first gently invite us to understand the world as something "alive” (artificially live). Such animation of the world will, paradoxically, make our outlook closer to that of pre-technological cultures which interpreted all aspects of nature as inhabited by teleological forces.

The second step will be a reconceptualization of our ontology in informational terms. It will become normal to consider the world as part of the infosphere, not so much in the dystopian sense expressed by a Matrix-like scenario, where the "real reality" is still as hard as the metal of the machines that inhabit it; but in the evolutionary, hybrid sense represented by an environment such as New Port City, the fictional, post-cybernetic metropolis of Ghost in the Shell. The infosphere will not be a virtual environment supported by a genuinely "material” world behind; rather, it will be the world itself that will be increasingly interpreted and understood informationally, as part of the infosphere. At the end of this shift, the infosphere will have moved 
from being a way to refer to the space of information to being synonymous with Being. This is the sort of informational metaphysics I suspect we shall find increasingly easy to embrace.

For the skeptic, there are plenty of daily examples that offer tangible evidence of such radical transformations. "RoboticCookware" is already available (http://www.vitacraft.com.nyud.net:8090/rfiq/home.html). MP3 players will soon be able to recommend new music to their users by learning from the tunes they (the users, that is) enjoyed (http://www.semanticaudio.com/). $\quad$ Your next fridge (http://www.lginternetfamily.co.uk/homenetwork.asp) will inherit your tastes and wishes from the old one, just as your new laptop can import your favourite settings from the old one; and it will interact with your new way of cooking and with the supermarket website, again, just as your laptop can talk to a printer or to another computer. We have all known that this was possible on paper for some time; the difference is that it is now actually happening in our kitchen.

As a consequence of such re-ontologization of our ordinary environment, we shall be living in an infosphere that will become increasingly synchronized (time), delocalised (space) and correlated (interactions). Previous revolutions (especially the agricultural and the industrial ones) created macroscopic transformation in our social structures and architectural environments, often without much foresight. The informational revolution is no less dramatic. We shall be in serious trouble, if we do not take seriously the fact that we are constructing the new environment that will be inhabited by future generations (Floridi and Sanders [2005]). We should be working on an ecology of the infosphere, if we wish to avoid problems such as a tragedy of the digital commons (Greco and Floridi [2004]). Unfortunately, I suspect it will take some time and a whole new kind of education and sensitivity to realise that the infosphere is a common space, which needs to be preserved to the advantage of all. One thing seems indubitable though: the digital divide will become a chasm, generating new forms of discrimination between those who can be denizens of the infosphere and those who cannot, between insiders and outsiders, between information rich and information poor. It will redesign the map of worldwide society, generating or widening generational, geographic, socio-economic and cultural divides. But the gap will not be reducible to the distance between industrialized and developing countries, since it will cut across societies. (Floridi [2002]). We are preparing the ground for tomorrow’s digital favelas. 


\section{The evolution of inforgs}

We have seen that we are probably the last generation to experience a clear difference between onlife and online. The third transformation that I wish to highlight concerns precisely the emergence of artificial and hybrid (multi)agents, i.e., partly artificial and partly human (consider, for example, a family as a single agent, equipped with digital cameras, laptops, palm pilots, iPods, mobiles, wireless network, digital TVs, DVDs, CD players, etc.).

These new agents already share the same ontology with their environment and can operate in it with much more freedom and control. We (shall) delegate or outsource to artificial agents memories, decisions, routine tasks and other activities in ways that will be increasingly integrated with us and with our understanding of what it means to be an agent. This is rather well known, but two other aspects of this transformation may be in need of some clarification.

On the one hand, in the re-ontologized infosphere, progressively populated by ontologically-equal agents, where there is no difference between processors and processed, online and offline, all interactions become equally digital. They are all interpretable as "read/write” (i.e., access/alter) activities, with “execute” the remaining type of process. It is easy to predict that, in such an environment, the moral status and accountability of artificial agents will become an ever more challenging issue (Floridi and Sanders [2004b]).

On the other hand, our understanding of ourselves as agents will also be deeply affected. I am not referring here to the sci-fi vision of a "cyborged” humanity. Walking around with something like a Bluetooth wireless headset implanted in your ear does not seem the best way forward, not least because it contradicts the social message it is also meant to be sending: being on call $24 \times 7$ is a form of slavery, and anyone so busy and important should have a PA instead. The truth is rather that being a sort of cyborg is not what people will embrace, but what they will try to avoid, unless it is inevitable (more on this shortly).

Nor am I referring to a GM humanity, in charge of its informational DNA and hence of its future embodiments. This is something that we shall probably see in the future, but it is still too far away, both technically (safely doable) and ethically (morally acceptable), to be discussed at this stage.

What I have in mind is a quieter, less sensational and yet crucial and profound change in our conception of what it means to be an agent. We are all becoming connected informational 
organisms (inforgs). This is happening not through some fanciful transformation in our body, but, more seriously and realistically, through the re-ontologization of our environment and of ourselves.

By re-ontologizing the infosphere, digital ICTs have brought to light the intrinsically informational nature of human agents. This is not equivalent to saying that people have digital alter egos, some Messrs Hydes represented by their @s, blogs and https. This trivial point only encourages us to mistake digital ICTs for merely enhancing technologies. The informational nature of agents should not be confused with a "data shadow"1 either. The more radical change, brought about by the re-ontologization of the infosphere, will be the disclosure of human agents as interconnected, informational organisms among other informational organisms and agents.

Consider the distinction between enhancing and augmenting appliances. The switches and dials of the former are interfaces meant to plug the appliance in to the user's body ergonomically. Drills and guns are perfect examples. It is the cyborg idea. The data and control panels of augmenting appliances are instead interfaces between different possible worlds: on the one hand there is the human user's Umwelt ${ }^{2}$, and on the other hand there are the dynamic, watery, soapy, hot and dark world of the dishwasher; the equally watery, soapy, hot and dark but also spinning world of the washing machine; or the still, aseptic, soapless, cold and potentially luminous world of the refrigerator. These robots can be successful because they have their environments "wrapped” and tailored around their capacities, not vice versa. Imagine someone trying to build a droid like C3PO capable of washing their dishes in the sink exactly in the same way as a human agent would. Now, ICTs are not augmenting or empowering in the sense just explained. They are re-ontologizing devices because they engineer environments that the user is then enabled to enter through (possibly friendly) gateways. It is a form of initiation. Looking at the history of the mouse (http://sloan.stanford.edu/mousesite/), for example, one discovers that our technology has not only adapted to, but also educated, us as users. Douglas Engelbart once told me that he had even experimented with a mouse to be placed under the desk, to be operated with one's leg, in order to leave the user’s hands free. HCI (Human-Computer Interaction) is a symmetric relation.

\footnotetext{
${ }^{1}$ The term is introduced by Westin [1968] to describe a digital profile generated from data concerning a user's habits online.

${ }^{2}$ The outer world, or reality, as it affects the agent inhabiting it.
} 
To return to our distinction, whilst a dishwasher interface is a panel through which the machine enters into the user's world, a digital interface is a gate through which a user can be (tele)present in the infosphere (Floridi [2005]). This simple but fundamental difference underlies the many spatial metaphors of “cyberspace”, "virtual reality”, "being online”, “surfing the web”, "gateway" and so forth. It follows that we are witnessing an epochal, unprecedented migration of humanity from its Umwelt to the infosphere itself, not least because the latter is absorbing the former. As a result, humans will be inforgs among other (possibly artificial) inforgs and agents operating in an environment that is friendlier to digital creatures. As digital immigrants like us are replaced by digital natives like our children, the latter will come to appreciate that there is no ontological difference between infosphere and Umwelt, only a difference of levels of abstractions (Floridi and Sanders [2004a]). And when the migration is complete, we shall increasingly feel deprived, excluded, handicapped or poor to the point of paralysis and psychological trauma whenever we are disconnected from the infosphere, like fish out of water.

One day, being an inforg will be so natural that any disruption in our normal flow of information will make us sick. Even literally. A simple illustration is provided by current BAN (Body Area Network) - systems “a base technology for permanent monitoring and logging of vital signs [...] [to supervise] the health status of patients suffering from chronic diseases, such as Diabetes and Asthma.” (http://www.ban.fraunhofer.de/index_e.html) - and Wearable Computers (http://www.businessweek.com/technology/content/mar2005/tc2005038_5955_tc119.htm ). Both phenomena are properly understood from an inforg (not a cyborg) perspective.

\section{Conclusion}

It would be useful to have some idea of what sort of empirical evidence we should look for that might signal the emergence of the infosphere as the real and only environment in which human inforgs will be living. How will we know that what has been predicted above is actually happening? By way of conclusion, here are five suggestions.

\section{1) Eight hours of life}

One important problem that we shall face will concern the availability of sufficient energy to stay connected to the infosphere non-stop. It is what Intel calls "the battery life challenge" (http://www.intel.com/products/centrino/enablingbatterylIfe.pdf). Today, we know that our 
autonomy is limited by the energy bottleneck of our batteries. The infosphere, and hence life as an inforg, will become a reality the closer we get to the 8 hours threshold.

\section{2) Google IRL}

You will know that ITentities have finally arrived when you will be able to use a search engine to find them in the house ("where are my glasses?”) or in the office (“where is my stapler?”) in the same way that you already locate a book in a library through its electronic catalogue. Google IRL (in real life) will signal the collapse of that thin membrane still separating the worlds of online and offline.

\section{3) Children of the PC}

For clear signs of digital migration in recent generations, some evidence can be gathered by looking at the evolution of the software game industry. For example, in the US, the average age of players is increasing, as the children of the post-computer revolutions are reaching their late thirties (http://www.theesa.com/files/2005EssentialFacts.pdf http://www.pacificepoch.com/uploads/docs/20060124_Sample_-

_Pacific_Epoch_Online_Game_Report_PreRelease.pdf). By the time they retire, in three or four decades, they will be living in the infosphere full-time.

\section{4) How do I know I am an inforg?}

If you spend more time connected than sleeping, you are an inforg. On average, Britons, for example, already spend more time online than watching TV (http://www.statistics.gov.uk/pdfdir/intacc0702.pdf).

\section{5) Virtual assets?}

One way of checking whether the new metaphysics has arrived is to look for the emergence of a serious economy of virtual assets. This involves two steps.

At the time of writing, End User License Agreements (EULA) of massively multiplayer online role-playing games (MMORPG) such as World of Warcraft still do not allow the sale of virtual assets. This would be like the EULA of MS-Word withholding from users the ownership of the digital documents created by means of the software. This is inevitably changing, as more people invest hundreds and then thousands of hours building their avatars. Future generations will inherit digital assets that they will want to own. Indeed, although it is forbidden, there are thousands of virtual assets on sale on e-bay. A quick check on the $14^{\text {th }}$ of March 2006 showed 
that the starting bid for a "World of Warcraft WTB rank14 or epic geared druid" was \$ 1500, a price much higher than the value of the average computer used to access that piece of information. Sony, more aggressively, already offers a "Station Exchange”, an official auction service that "provides players a secure method of buying and selling [in dollars, my specification] the right to use in game coin, items and characters in accordance with SOE's license agreement, rules and guidelines” (http://stationexchange.station.sony.com/).

Again, for the skeptical reader, a comparison and some hard evidence might be useful. In recent years, many countries have followed the US in counting acquisition of software not as a current business expense but as an investment, to be treated as any other capital input that is repeatedly used in production over time (The Economist [Feb 16th 2006]). This has meant that spending on software now regularly contributes to GDPs. So software is acknowledged to be a (digital) good, even if somewhat intangible. It should not be too difficult to accept that virtual assets too may represent important investments. As for the hard evidence, the phenomenon of socalled "virtual sweatshops" in China is very indicative. In claustrophobic and overcrowded rooms, workers play online games, like World of Warcraft or Lineage, for up to twelve hours a day, to create virtual goods, such as characters, equipments or in-game currency, which can then be sold to other players (warning: the following video is rather disturbing http://youtube.com/watch?v=ho5Yxe6UVv4).

Once ownership of virtual assets has been legally established, the second step will be to check for the emergence of property litigations (already happening: in May 2006 a Pennsylvania lawyer sued the publisher of Second Life for allegedly having unfairly confiscated tens of thousands of dollars worth of his virtual land and other property http://www.wired.com/news/culture/0,70909-0.html) and insurance that provides protection against risks to them. It won't be a revolution in business, but it might be comparable to the pet insurances you can currently buy at your local supermarket (http://www.sainsburysbank.co.uk/insuring/ins_petinsurance_pet_skip.shtml?source=NETGOO GLPETIEM010001). Again, World of Warcraft provides an excellent example. The six million players (as of the $1^{\text {st }}$ of August, 2006, this is larger than the whole population of Norway, for example) who (will) have spent billions of man-hours constructing, enriching and refining their 
avatars will be more than willing to spend a few dollars to insure them. In the near future, this will look normal.

\section{Acknowledgements}

I am grateful to Adam C. Engst for having prompted me to write this paper and to Gian Maria Greco, Ken Herold, Gianluca Paronitti, Sebastian Sequoiah-Grayson, Miguel Sicart and Matteo Turilli for discussions of several ideas concerning Ghost in the Shell and online games during our meetings. Paul Oldfield kindly checked the last draft.

\section{References}

Floridi, L. 1996, "Internet: Which Future for Organized Knowledge, Frankenstein or Pygmalion?" The Information Society, 9, 5-16.

Floridi, L. 2002, "Information Ethics: An Environmental Approach to the Digital Divide", Philosophy in the Contemporary World, 9(1), 39-45.

Floridi, L. 2005, "Presence: From Epistemic Failure to Successful Observability", Presence: Teleoperators and Virtual Environments, 14(6), 656-667.

Floridi, L., and Sanders, J. W. 2001, "Artificial Evil and the Foundation of Computer Ethics", Ethics and Information Technology, 3(1), 55-66.

Floridi, L., and Sanders, J. W. 2004a, "The Method of Abstraction" in Yearbook of the Artificial. Nature, Culture and Technology. Models in Contemporary Sciences, edition, edited by M. Negrotti (Bern: Peter Lang), 177-220.

Floridi, L., and Sanders, J. W. 2004b, "On the Morality of Artificial Agents", Minds and Machines, 14(3), 349-379.

Floridi, L., and Sanders, J. W. 2005, "Internet Ethics: The Constructionist Values of Homo Poieticus" in The Impact of the Internet on Our Moral Lives, edition, edited by Robert Cavalier (New York: SUNY),

Greco, G. M., and Floridi, L. 2004, "The Tragedy of the Digital Commons", Ethics and Information Technology, 6(2), 73-82.

Lyman, P., and Varian, H. R. 2003, How Much Information?, http://www.sims.berkeley.edu/research/projects/how-much-info2003/execsum.htm\#summary,

Mellor, C. April 06, 04, "Google's Storage Strategy", TechWorld. http://www.techworld.com/opsys/features/index.cfm?fuseaction=displayfeatures\&feature id=467\&page $=1$ \&pagepos $=6$

Microsoft Research 2005, "The Towards 2020 Science". http://research.microsoft.com/towards2020science/background_overview.htm

Nature 2006, "2020 - Future of Computing", 440.

O'Reilly, T. 2005, What Is Web 2.0 - Design Patterns and Business Models for the Next Generation of Software, http://www.oreillynet.com/pub/a/oreilly/tim/news/2005/09/30/what-is-web-20.html,

The Economist Feb 16th 2006, "Software Investment - Now They See It".

Westin, A. F. 1968, Privacy and Freedom 1st (New York: Atheneum). 